\title{
INVARIANTS OF EUCLIDEAN REFLECTION GROUPS
}

\author{
BY \\ LOUIS SOLOMON(1)
}

1. Introduction and statement of results. Let $\boldsymbol{R}^{n}$ be $n$-dimensional Euclidean space and let $G$ be a finite group of orthogonal transformations of $\boldsymbol{R}^{n}$ generated by reflections. Let $V=C^{n}$ be the complexification of $R^{n}$. Then $G$ acts naturally in $V$ and we say that $G$ is a Euclidean reflection group in $V$. Let $S$ be the $C$-algebra of complex-valued polynomial functions on $V$, let $I(S)$ be the subalgebra of polynomials invariant under $G$ and let $F$ be the ideal of $S$ generated by the homogeneous elements of positive degree in $I(S)$. Chevalley [2] has proved that

(a) $I(S)$ is generated over $C$ by $n$ algebraically independent homogeneous polynomials $f_{1}, \cdots, f_{n}$ and the unit element.

(b) $S / F$ as $G$-module affords the regular representation of $G$.

In view of (b) every irreducible $G$-module $M$ occurs in $S / F$ with multiplicity equal to $\operatorname{dim} M$. Since $F$ is a homogeneous ideal, $S / F=\Sigma_{q}(S / F)_{q}$ is naturally graded. We prove two theorems concerning the graded $G$-module structure of $S / F$.

The symmetric group $G$ of degree $n$ acts naturally as a Euclidean reflection group in $V$ by permuting the elements of a basis. The irreducible characters of $G$ are in 1-1 correspondence with partition diagrams of $n$ nodes [6]. In the set of partition diagrams there is a natural ordering. The evidence suggests that characters which occur early in this ordering occur early in the decomposition $S / F=\Sigma_{q}(S / F)_{q}$ in the sense that they occur for small values of $q$. On the other hand, a formula of Frobenius [6, p. 534] indicates that if a character occurs early in the partition ordering, then a reflection (transposition) fixes a large part of the corresponding representation space. These observations led to the following.

THEOREM 1. Let $G$ be a Euclidean reflection group and let $M$ be an irreducible G-module. Let $\gamma \in G$ be a reflection, let $M_{\gamma}^{-}$be the subspace of all $x \in M$ such that $\gamma x=-x$ and suppose that $\operatorname{dim} M_{\gamma}^{-}=\operatorname{dim} M^{-}$is independent of $\gamma$. If $M$ is an irreducible constituent of $(S / F)_{q}$ for precisely the values $q_{1}(M), \cdots, q_{l}(M)$, $l=\operatorname{dim} M$, then the average of the $q_{i}(M)$ is

$$
\frac{\operatorname{dim} M^{-}}{\operatorname{dim} M} r
$$

where $r$ is the number of reflections in $G$.

Received by the editors June 7, 1963.

(1) This research was supported by the National Science Foundation under grant G-21514 
The assumption that $\operatorname{dim} M_{\gamma}^{-}$is independent of $\gamma$ is satisfied for all $M$ when the reflections in $G$ form a single conjugate class, and is satisfied for the irreducible modules $E_{p}$ of Theorem 2 when $G$ is a Weyl group. The extreme cases in Theorem 1 are given by $\operatorname{dim} M^{-}=0$, corresponding to the principal character, and $\operatorname{dim} M^{-}=\operatorname{dim} M$, corresponding to the alternating character of $G$.

THEOREM 2. Let $L$ be a complex simple Lie algebra and let $V$ be a Cartan subalgebra of L. Let $G$ be the Weyl group of L acting in $V$. Let $E_{p}$ be the Gmodule of alternating multilinear p-forms over $V$. Then $E_{p}$ is irreducible and occurs as a constituent of $(S / F)_{q}$ for precisely the values $q=m_{i_{1}}+\cdots+m_{i_{n}}$, $i_{1}<\cdots<i_{p}$, where $m_{i}+1$ is the degree of $f_{i}$. A basis for the isotypic component of $S / F$ of type $E_{p}$ is given by the set of minors of order $p$ of the Jacobian matrix $J$ of $f_{1}, \cdots, f_{n}$ reduced $\bmod F$.

The irreducibility of the modules $E_{p}$ for Weyl groups of the exceptional Lie algebras $\mathbb{E}_{6}, \mathbb{E}_{7}$ was noticed by Frame [5]. It seems likely that Theorem 2 is true for all the Euclidean reflection groups. The proof we give depends on a theorem of Burnside [1] on Weyl groups which allows us to compute certain invariants of G. $\left({ }^{2}\right)$ Theorem 2 and its proof have the following corollaries:

(2a) All minors of $J$ are linearly independent over $C$ and remain linearly independent after reduction $\bmod F$. In particular, none of them vanish.

(2b) The algebra of invariants $I(S / F \otimes E)$ is an exterior algebra over $C$ on $n$ generators.

(2c) If $E=\Sigma_{p} E_{p}$ is the Grassmann algebra of $V$, then the algebra of invariants $I(E \otimes E)$ is a truncated polynomial algebra over $C$, generated by the unit and an element $w$ such that $w^{n+1}=0$. The generator $w$ may be identified with the Killing form.

(2d) For each $p=0, \cdots, n$ there exists a homogeneous isomorphism

$$
I\left(S / F \otimes E_{p}\right) \simeq I\left(S / F \otimes E_{n-p}\right)
$$

of graded vector spaces. Existence of this isomorphism for $p=1$ is equivalent to so-called double duality in the exponents $m_{i}$, the fact that if the $m_{i}$ are arranged in increasing order $m_{1} \leqq \cdots \leqq m_{n}$ then $m_{i}+m_{n-i+1}$ is independent of $i$.

The double duality in the exponents $m_{i}$ was a long standing mystery for Weyl groups, explained a few years ago by Coleman [3] and Kostant [7]. Even if one assumes the double duality as known, the argument in (2d) does not furnish an explicit isomorphism $I\left(S / F \otimes E_{1}\right) \simeq I\left(S / F \otimes E_{n-1}\right)$. We prove the existence of the isomorphism by computing the Poincaré series of both spaces. It would thus be interesting to give a direct invariant-theoretic proof for the double duality by exhibiting an isomorphism which is in some sense a natural one. We have not been able to do this, but present a line of argument which seems to lead in the right

(2) Added in proof. R. Steinberg has kindly shown me a proof of irreducibility which is independent of Burnside's theorem. His argument is valid for all the Euclidean groups. 
direction. The elements of $S \otimes E_{1}$ may be viewed as differential 1-forms on $V$. We study the space of those differential 1 -forms on $V$ which are skew invariant under $G$, in the sense that they are invariant under the rotation subgroup $H$ of $G$ and change sign under the elements outside $H$. It is not hard to show that this space is a free module over $I(S)$ of rank $n$. From the double duality and the fact that $G$ has a unique invariant quadratic form one concludes that among the polynomials $f_{1}, \cdots, f_{n}$ there is a unique polynomial $f_{n}$ of greatest degree. Then assuming the double duality we prove the following.

THEOREM 3. Let $G$ be an irreducible Euclidean reflection group in $V$. Choose coordinates in $V$ and let $u_{1}, \cdots, u_{n}$ be the minors of order $n-1$ of $J$ obtained by deleting the partial derivatives of $f_{n}$. If $u_{1}, \cdots, u_{n}$ are algebraically independent, then there exists a homogeneous derivation $\hat{d}: S \rightarrow S \otimes E_{1}$ of $S$-modules such that $\hat{d} f_{1}, \cdots, \hat{d} f_{n}$ are a basis for the module of skew invariant differential 1-forms over $I(S)$.

Granted the existence of the map $\hat{d}$, we can construct an explicit isomorphism $I\left(S / F \otimes E_{1}\right) \simeq I\left(S / F \otimes E_{n-1}\right)$. We have been able to verify the algebraic independence of $u_{1}, \cdots, u_{n}$ in special cases but have no general argument. If the $u_{i}$ are algebraically independent, then the Jacobian (determinant) of the $u_{i}$ must be a constant multiple of $(\operatorname{det} J)^{n-2}$.

We work over the complex field $C$ as a matter of convenience, and irreducibility of modules will mean irreducibility over $\boldsymbol{C}$. The complex field is probably an alien here because a likely conjecture of Kostant states that all the absolutely irreducible representations of a Euclidean reflection group may be written with coefficients in $\boldsymbol{R}$. In any case, a real linear group which contains a reflection and is irreducible over $\boldsymbol{R}$ remains irreducible over $\boldsymbol{C}$.

2. Notation. In this section we introduce some notation and collect some elementary facts about invariants and characters. Let $G$ be a finite group of order $g$. By a graded $G$-module we mean a $G$-module which is a graded vector space $M=\sum_{q \geqq 0} M_{q}$ over $C$, in which each homogeneous component $M_{q}$ is a $G$-module finite dimensional over $C$. Let $\mu_{q}$ be the character of $G$ corresponding to the module $M_{q}$. To the graded $G$-module $M$ we let correspond the series

$$
M(t, \gamma)=\sum_{q \geqq 0} \mu_{q}(\gamma) t^{q}, \quad \gamma \in G
$$

For $\gamma=1$ this becomes the Poincaré series

$$
M(t)=\sum_{q \geqq 0}\left(\operatorname{dim} M_{q}\right) t^{q}
$$

of the graded vector space $M$. All the tensor products we consider are tensor products over $C$. If $M, N$ are graded $G$-modules, then $M \otimes N$ has a natural structure of graded $G$-module with the grading and $G$-module structure defined by 


$$
\begin{aligned}
(M \otimes N)_{q} & =\sum_{a+b=q} M_{a} \otimes N_{b}, \\
\gamma(x \otimes y) & =\gamma x \otimes \gamma y, \quad x \in M, y \in N, \gamma \in G .
\end{aligned}
$$

From the fact that the character of a tensor product (direct sum) of two $G$-modules is the product (sum) of the characters it follows that

$$
(M \otimes N)(t, \gamma)=M(t, \gamma) N(t, \gamma) .
$$

We let $I(M)$ denote the submodule of invariants of $M$, elements $x \in M$ such that $\gamma x=x$ for all $\gamma \in G$. For a finite dimensional $M$ with character $\mu$ the connection between invariants and characters is the formula

$$
\operatorname{dim} I(M)=\frac{1}{g} \sum_{\gamma \in G} \mu(\gamma) .
$$

Thus for a graded $M$ we have

$$
I(M)(t)=\frac{1}{g} \sum_{\gamma \in G} M(t, \gamma) .
$$

The dual $M^{*}$ of $M$ has a natural $G$-module structure defined by

$$
(\gamma f)(x)=f\left(\gamma^{-1} x\right), \quad x \in M, f \in M^{*}, \gamma \in G,
$$

and we may extend this action to the algebra of polynomial functions on $M$ or the Grassmann algebra of $M$. If $\mu$ is the character of $M$ then $\mu^{*}(\gamma)=\mu\left(\gamma^{-1}\right)$ is the character of $M^{*}$. We have an isomorphism $M \cong M^{*}$ of $G$-modules if and only if the character of $M$ is real. The space $\operatorname{Hom}_{\mathrm{C}}(M, N)$ has a natural $G$-module structure defined by

$$
(\gamma \phi)(x)=\gamma\left(\phi\left(\gamma^{-1} x\right)\right), \quad x \in M, \phi \in \operatorname{Hom}_{\mathbf{C}}(M, N), \gamma \in G,
$$

and the submodule $I\left(\operatorname{Hom}_{\mathrm{C}}(M, N)\right)$ is just the space $\operatorname{Hom}_{G}(M, N)$ of $G$ module homomorphisms. The natural isomorphism of vector spaces $\operatorname{Hom}_{\mathrm{C}}(M, N) \simeq N \otimes M^{*}$ is an isomorphism of $G$-modules and induces an isomorphism $\operatorname{Hom}_{G}(M, N) \simeq I\left(N \otimes M^{*}\right)$. In particular we see that if $M$ is irreducible then $\operatorname{dim} I\left(N \otimes M^{*}\right)$ is the multiplicity of $M$ in $N$, and that $M$ is irreducible if and only if $\operatorname{dim} I\left(M \otimes M^{*}\right)=1$.

3. Let $G$ be a Euclidean reflection group in $V$ and let $S$ be the algebra of complex valued polynomial functions on $V$. Then $S=\Sigma_{q \geqq 0} S_{q}$ has a natural structure of graded $G$-module. A formula of Molien, easy to verify by assuming $\gamma$ in diagonal form, states that

$$
S\left(t, \gamma^{-1}\right)=\frac{1}{\left(1-\omega_{1}(\gamma) t\right) \cdots\left(1-\omega_{n}(\gamma) t\right)}
$$

where $\omega_{1}(\gamma), \cdots, \omega_{n}(\gamma)$ are the eigenvalues of $\gamma$ as linear transformation of $V$. 
If $M$ is a finite dimensional $G$-module we give $S \otimes M$ the grading defined by $(S \otimes M)_{q}=S_{q} \otimes M$. Then Molien's formula implies

$$
I(S \otimes M)(t)=\frac{1}{g} \sum_{\gamma \in G} \frac{\mu\left(\gamma^{-1}\right)}{\left(1-\omega_{1}(\gamma) t\right) \cdots\left(1-\omega_{n}(\gamma) t\right)}
$$

where $\mu$ is the character of $M$. From Theorem (a) of Chevalley we see that

$$
I(S)(t)=\frac{1}{\left(1-t^{m_{1}+1}\right) \cdots\left(1-t^{m_{n}+1}\right)} .
$$

Chevalley has also shown [2] that if $p_{1}, \cdots, p_{k} \in S$ form a $C$-basis for $S / F$ when reduced $\bmod F$, then $p_{1}, \cdots, p_{k}$ are a basis for $S$ as free module over $I(S)$. From this fact we readily deduce the following two lemmas.

LEMma 1. Let $\tau_{q}$ be the character of $(S / F)_{q}$. Then

$$
\sum_{q} \tau_{q}\left(\gamma^{-1}\right) t^{q}=\frac{\left(1-t^{m_{1}+1}\right) \cdots\left(1-t^{m_{n}+1}\right)}{\left(1-\omega_{1}(\gamma) t\right) \cdots\left(1-\omega_{n}(\gamma) t\right)} .
$$

Proof. Let $p_{1}, \cdots, p_{k} \in S$ form a $C$-basis for $S / F$ when reduced $\bmod F$. Then the map $\sum_{i} s_{i} p_{i} \rightarrow \sum_{i} s_{i} \otimes\left(p_{i}+F\right), s_{i} \in I(S)$ defines an isomorphism $S \simeq I(S) \otimes S / F$ of graded $G$-modules. Since $G$ acts trivially on $I(S)$ we have $S(t, \gamma)=$ $I(S)(t)(S / F)(t, \gamma)$. Thus from (3.1) and (3.3)

$$
(S / F)\left(t, \gamma^{-1}\right)=\frac{\left(1-t^{m_{1}+1}\right) \cdots\left(1-t^{m_{n}+1}\right)}{\left(1-\omega_{1}(\gamma) t\right) \cdots\left(1-\omega_{n}(\gamma) t\right)}
$$

which proves the lemma.

If we let $t \rightarrow 1$ we find $\Sigma_{q} \tau_{q}(\gamma)=0$ if $\gamma \neq 1$ and $\Sigma_{q} \tau_{q}(1)=g$, so that $\Sigma_{q} \tau_{q}$ is the character of the regular representation of $G$. Thus $S / F$ affords the regular representation of $G$. If $M$ is an irreducible $G$-module we let $a_{q}(M)$ be the multiplicity of $M$ in $(S / F)_{q}$. Since $S / F$ contains $M$ with multiplicity equal to $\operatorname{dim} M$ we have

$$
\sum_{q} a_{q}(M)=\operatorname{dim} M \text {. }
$$

We view $S \otimes M$ naturally as an $S$-module and then $I(S \otimes M)$ is an $I(S)$-module.

LemMA 2. Let $M$ be an irreducible $G$-module. Then $I(S \otimes M)$ is a free module over $I(S)$. It has a basis over I $(S)$ consisting of homogeneous elements in which the number of elements of degree $q$ is $a_{q}\left(M^{*}\right)$. The rank of $I(S \otimes M)$ as $I(S)$-module is equal to $\operatorname{dim} M$.

Proof. In the proof of Lemma 1 we have remarked that $S \simeq I(S) \otimes(S / F)$ and hence $S \otimes M \simeq I(S) \otimes(S / F) \otimes M$. Since $G$ acts trivially on $I(S)$ we see by averaging over the group that $I(S \otimes M) \simeq I(S) \otimes I(S / F \otimes M)$. Thus $I(S \otimes M)$ is free over $I(S)$ and we may choose as basisa $C$-basis for $I(S / F \otimes M)$. This may be 
chosen as a union of $C$-bases for the $I\left((S / F)_{q} \otimes M\right)$. But $\operatorname{dim} I\left((S / F)_{q} \otimes M\right)$ is the multiplicity of the irreducible $M^{*}$ in $(S / F)_{q}$ so $\operatorname{dim} I\left((S / F)_{q} \otimes M\right)=a_{q}\left(M^{*}\right)$. The rank of $I(S \otimes M)$ as $I(S)$-module is thus $\Sigma_{q} a_{q}\left(M^{*}\right)=\operatorname{dim} M^{*}=\operatorname{dim} M$. The argument shows that $I(S \otimes M)$ is free over $I(S)$ for any $G$-module $M$.

4. To prove Theorem 1 we simply compute the Poincaré series $I\left(S \otimes M^{*}\right)(t)$ in two ways and compare the results for $t=1$. Set $a(t)=\Sigma_{q} a_{q}(M) t^{q}$. From Lemma 2 with $M$ replaced by $M^{*}$ we have

$$
I\left(S \otimes M^{*}\right)(t)=\frac{a(t)}{\left(1-t^{m_{1}+1}\right) \cdots\left(1-t^{m_{n}+1}\right)}
$$

and thus from (3.2) we see that

$$
\frac{1}{g} \sum_{\gamma \in G} \frac{\mu(\gamma)}{\left(1-\omega_{1}(\gamma) t\right) \cdots\left(1-\omega_{n}(\gamma) t\right)}=\frac{a(t)}{\left(1-t^{m_{1}+1}\right) \cdots\left(1-t^{m_{n}+1}\right)}
$$

where $\mu$ is the character of $M$. Let $G_{1}$ be the set of elements of $G$, distinct from the identity, which fix an $n-1$ dimensional subspace of $V$. For $\gamma \in G_{1}$ the eigenvalues $\omega_{i}(\gamma)$ are $1,1, \cdots, 1, \omega$ where $\omega$ is a root of unity. Now the fact that $G$ may be written as a real orthogonal group implies $\omega=-1$. The left-hand side of (4.1) becomes

$$
\frac{1}{g}\left[\frac{\mu(1)}{(1-t)^{n}}+\frac{1}{(1-t)^{n-1}(1+t)} \sum_{\gamma \in G_{1}} \mu(\gamma)+\cdots\right]
$$

where $\cdots$ denotes terms which have at most $(1-t)^{n-2}$ in the denominator. Since $a(1)=\Sigma_{q} a_{q}(M)=\operatorname{dim} M=\mu(1)$ we have

$$
\frac{1}{(1-t)^{n-1}(1+t)} \sum_{\gamma \in G_{1}} \mu(\gamma)+\cdots=\frac{g a(t)}{\left(1-t^{m_{1}+1}\right) \cdots\left(1-t^{m_{n}+1}\right)}-\frac{a(1)}{(1-t)^{n}} .
$$

Now multiply both sides by $(1-t)^{n-1}$ and let $t \rightarrow 1$. Using the known formula $[4 ; 8] g=\prod_{i}\left(m_{i}+1\right)$ for the group order we find

$$
\frac{1}{2} \sum_{\gamma \in G_{1}} \mu(\gamma)=-a^{\prime}(1)+\frac{1}{2}\left(\sum_{i} m_{i}\right) a(1)
$$

where $a^{\prime}$ denotes the derivative with respect to $t$. For $\gamma \in G_{1}, \mu(\gamma)=\operatorname{dim} M_{\gamma}^{+}$ $-\operatorname{dim} M_{\gamma}^{-}$is, by assumption, independent of $\gamma$. The number of elements in $G_{1}$ is the number of reflections in $G$ which is known $[4 ; 8]$ to be $\sum_{i} m_{i}$. From the definition of $a(t)$ we have $a^{\prime}(1)=\Sigma_{q} q a_{q}(M)$. If we insert this information in (4.2) we find

$$
\frac{1}{2}\left(\sum_{i} m_{i}\right)\left(\operatorname{dim} M^{+}-\operatorname{dim} M^{-}\right)=-\sum_{q} q a_{q}(M)+\frac{1}{2}\left(\sum_{i} m_{i}\right)\left(\operatorname{dim} M^{+}+\operatorname{dim} M^{-}\right)
$$


so that

$$
\sum_{q} q a_{q}(M)=\left(\sum_{i} m_{i}\right) \operatorname{dim} M^{-}
$$

The average of the $q_{i}(M)$ is then

$$
\frac{\sum_{q} q a_{q}(M)}{\sum_{q} a_{q}(M)}=\frac{\operatorname{dim} M^{-}}{\operatorname{dim} M} r
$$

with $r=\sum_{i} m_{i}$. The significance of the integer $r=\sum_{i} m_{i}$ in this formula becomes clear if one observes, with Lemma 1 , that $r$ is the largest integer $q$ for which $(S / F)_{q} \neq 0$.

5. To prove Theorem 2 we shall need some results from an earlier article [9] together with a lemma concerning certain invariants of the symmetric group. Let $E=\Sigma_{p} E_{p}$ be the Grassman algebra of $V$. The homogeneous component $E_{p}$ of degree $p$ is the space of all $p$-linear alternating functions on $V$. We identify $E_{0}$ with $C$ and $E_{1}$, as vector space, with $S_{1}$. The group $G$ acts naturally on $E$ and on $S \otimes E$. Choose a coordinate system $x_{1}, \cdots, x_{n}$ in $V$ and let $d: S \otimes E \rightarrow S \otimes E$ be the $C$-linear map defined by

$$
d: s \otimes x_{i_{1}} \wedge \cdots \wedge x_{i_{n}} \rightarrow \sum_{j=1}^{n} \frac{\partial s}{\partial x_{j}} \otimes x_{j} \wedge x_{i_{1}} \wedge \cdots \wedge x_{i_{n}}, \quad s \in S .
$$

If we identify $S$ with $S \otimes C$ then $d x_{i}=d\left(x_{i} \otimes 1\right)=1 \otimes x_{i}$ so that the elements of $S \otimes E_{p}$ may be written in the form

$$
\sum_{i_{1}<\cdots<i_{n}} s_{i_{1}} \cdots_{i_{n}} d x_{i_{1}} \cdots d x_{i_{n}}, \quad s_{i_{1}} \cdots_{i_{n}} \in S .
$$

It is clear that $S \otimes E$ is just the algebra of differential forms on $V$ and that $d$ is exterior differentiation. Since $d$ commutes with the action of $G$ on $S \otimes E$ it follows that $d$ maps $I(S \otimes E)$ into $I(S \otimes E)$. In particular, the differentials $d f_{i}$ are invariants of $S \otimes E$. We have shown in [9] that the $C$-algebra $I(S \otimes E)$ of invariant differential forms is an exterior algebra on $n$ generators over the $C$-algebra $I(S)$ of invariant polynomials, and is in fact generated over $I(S)$ by the differentials $d f_{1}, \cdots, d f_{n}$ of the polynomial invariants $f_{1}, \cdots, f_{n}$ and the unit element. It follows that

$$
I\left(S \otimes E_{p}\right)(t)=\frac{\sigma_{p}\left(t^{m_{1}}, \cdots, t^{m_{n}}\right)}{\left(1-t^{m_{1}+1}\right) \cdots\left(1-t^{m_{n}}+1\right)}, \quad p=1, \cdots, n,
$$

where $\sigma_{p}\left(t_{1}, \cdots, t_{n}\right)$ is the $p$ th elementary symmetric function in the indeterminates $t_{1}, \cdots, t_{n}$.

LEMMA 3. Let $x_{1}, \cdots, x_{n}$ be indeterminates, let $G$ be the symmetric group on $x_{1}, \cdots, x_{n}$ and let $E$ be the exterior algebra on $x_{1}, \cdots, x_{n}$ over $C$. The group $G$ acts naturally on the commutative algebra $E \otimes E$. Set 


$$
u=\sum_{i} x_{i} \otimes x_{i}, \quad v=\sum_{i<k} x_{i} \otimes x_{k}+x_{k} \otimes x_{i} .
$$

Then

$$
I\left(E_{p} \otimes E_{p}\right)=C u^{p} \oplus C u^{p-1} v, \quad p=1, \cdots, n-1 .
$$

Proof. Clearly both $u^{p}$ and $u^{p-1} v$ are in $I\left(E_{p} \otimes E_{p}\right)$. To hold the indices in check we let $\Omega$ denote the set of increasing sequences $i_{1}<\cdots<i_{p}$ of $p$ integers chosen from $1, \cdots, n$, we let $(i)$ denote an element of $\Omega$, let $\{i\}$ be the corresponding unordered set, and write $x_{(i)}=x_{i_{1}} \wedge \cdots \wedge x_{i_{n}}$. Suppose $y=\sum c_{(i),(k)} x_{(i)} \otimes x_{(k)} \in I\left(E_{p} \otimes E_{p}\right)$ where $c_{(i),(k)} \in C$ and the sum is over all pairs $(i),(k)$ of elements of $\Omega$. If for given sets $\{i\},\{k\}$ the intersection $\{i\} \cap\{k\}$ contains fewer than $p-1$ elements, then there exist two distinct indices, say $k_{\alpha}, k_{\beta}$, which are distinct from all elements of $\{i\}$. Now apply the transposition $\left(k_{\alpha} k_{\beta}\right)$ of $G$ to each term in the sum $y$. The invariance of $y$ shows that $c_{(i),(k)}=-c_{(i),(k)}$ and hence $c_{(i),(k)}=0$. Thus we may write $y=y_{1}+y_{2}$ where $y_{1}$ is a linear combination of elements $x_{(i)} \otimes x_{(k)}$ such that $\{i\} \cap\{k\}$ contains $p$ elements, in other words $\{i\}=\{k\}$, and $y_{2}$ is a linear combination of elements $x_{(i)} \otimes x_{(k)}$ such that $\{i\} \cap\{k\}$ contains $p-1$ elements. Invariance of $y$ implies the invariance of $y_{1}$ and $y_{2}$. We thus have $y_{1}=\sum_{(i)} b_{(i)} x_{(i)} \otimes x_{(i)}$ with $b_{(i)} \in C$ and invariance of $y_{1}$ shows that all the $b_{(i)}$ are equal, say $b_{(i)}=b$. Then $y_{1}=b \sum_{(i)} x_{(i)} \otimes x_{(i)}$ is a $C$-multiple of $u^{p}=p ! \sum_{(i)} x_{(i)} \otimes x_{(i)} \cdot$ Similarly with slightly more effort one sees that $y_{2}$ is a $C$-multiple of $u^{p-1} v$. Thus the elements $u^{p}, u^{p-1} v$ span $I\left(E^{p} \otimes E^{p}\right)$. For $p<n$ both $u^{p}$ and $u^{p-1} v$ are not zero and hence linearly independent over $C$. This proves the lemma. The argument breaks down for $p=n$ only because $u^{n-1} v=0$ and in that case we have $I\left(E_{n} \otimes E_{n}\right)=C u^{n}$. The elements $u, v$ also satisfy the relations $u^{n+1}=0$ and $v^{2}=0$.

We are now in position to prove Theorem 2. Since $L$ is simple, $G$ acting in $V$ is an irreducible group. A theorem of Burnside [1] states that there exists a coordinate system $x_{1}, \cdots, x_{n}$ in $V$ such that $G$ acting on $V^{*} \simeq E_{1}$ includes the symmetric group $H$ on $x_{1}, \cdots, x_{n}$. In this coordinate system the Killing form must be

$$
\frac{a}{2} \sum_{i} x_{i}^{2}+b \sum_{i<k} x_{i} x_{k} \in I\left(S_{2}\right)
$$

where $a, b$ are real numbers. We cannot have $a=0$ because the form is positive definite. We let $I(E \otimes E)$ denote the elements of $E \otimes E$ invariant under $G$ and $I_{H}(E \otimes E)$ the elements invariant under $H$. Under the map $f \rightarrow \Sigma_{i}\left(\partial f / \partial x_{i}\right) \otimes x_{i}$ of $S_{2} \rightarrow E_{1} \otimes E_{1}$ the Killing form maps into $a u+b v \in I\left(E_{1} \otimes E_{1}\right)$ where $u, v \in I_{H}\left(E_{1} \otimes E_{1}\right)$ are the invariants of Lemma 3. Since $I(E \otimes E) \subseteq I_{H}(E \otimes E)$, Lemma 3 shows that $\operatorname{dim} I\left(E_{p} \otimes E_{p}\right) \leqq 2$. Suppose $\operatorname{dim} I\left(E_{p} \otimes E_{p}\right)=2$ for some $p=1, \cdots, n-1$. We prove that $\operatorname{dim} I\left(E_{n-1} \otimes E_{n-1}\right)=2$. If $p=n-1$ there is nothing to prove so assume $p<n-1$. From Lemma 3 we see that $u^{p} \in I\left(E_{p} \otimes E_{p}\right)$ and $u^{p-1} v \in I\left(E_{p} \otimes E_{p}\right)$. Since $v^{2}=0$ it follows that both $a u^{p} v=u^{p-1} v(a u+b v)$ and $a u^{p+1}+b u^{p} v=u^{p}(a u+b v)$ are in $I\left(E_{p+1} \otimes E_{p+1}\right)$. Since $p<n-1$ we 
have $u^{p} v \neq 0$ and since $a \neq 0$ it follows that $\operatorname{dim} I\left(E_{p+1} \otimes E_{p+1}\right)=2$. We conclude by induction that $\operatorname{dim} I\left(E_{n-1} \otimes E_{n-1}\right)=2$. Let $Z$ be the 1-dimensional $G$-module defined by the homomorphism $\gamma \rightarrow \operatorname{det} \gamma, \gamma \in G$. Then $E_{n-1} \simeq E_{1} \otimes Z$ as $G$-modules. Since $\operatorname{det} \gamma= \pm 1, Z \otimes Z \simeq C$ is the trivial $G$-module. Then $E_{n-1} \otimes E_{n-1} \simeq E_{1} \otimes E_{1}$ as $G$-modules so that $\operatorname{dim} I\left(E_{1} \otimes E_{1}\right)=2$ which contradicts the irreducibility of $E_{1}$. Thus $\operatorname{dim} I\left(E_{p} \otimes E_{p}\right)=1$ for all $p$ and hence $E_{p}$ is irreducible for all $p$.

Let $\theta: S \rightarrow S / F$ be the natural map and extend $\theta$ to a map, denoted again $\theta$, of $S \otimes E \rightarrow S / F \otimes E$ by letting it be the identity on $E$. Then $\theta$ is a homomorphism of $G$-modules and of $C$-algebras. Since $\theta$ is a homomorphism of $G$-modules we certainly have $\theta I(S \otimes E) \subseteq I(S / F \otimes E)$. Actually we have $\theta I(S \otimes E)=I(S / F \otimes E)$ because, using complete reducibility of the representations of $G$, we may choose a graded $G$-module $T$ such that $S=F \oplus T$ and then $\theta: I(T \otimes E) \rightarrow I(S / F \otimes E)$ is an isomorphism. Set $z_{i}=\theta\left(d f_{i}\right)$. Since $I(S \otimes E)$ is generated over $I(S)$ by the $d f_{i}$ and the unit element, and since every element $s \in I(S)$ may be written as $s=s_{0}+s_{1}$ with $s_{0} \in C$ and $s_{1} \in F$, it follows that the $z_{i}$ together with the unit element generate $I(S / F \otimes E)$ as algebra over $C$. Thus the $\left(\begin{array}{l}n \\ p\end{array}\right)$ elements $z_{i_{1}} \cdots z_{i}$ $i_{1}<\cdots<i_{p}$ generate $I\left(S / F \otimes E_{p}\right)$ as vector space over $C$. In fact they form a basisfor $I\left(S / F \otimes E_{p}\right)$ because Lemma 2 shows that $\operatorname{dim} I\left(S / F \otimes E_{p}\right)=\operatorname{dim} E_{p}=\left(\begin{array}{c}n \\ p\end{array}\right)$. Thus the $z_{i_{1}} \cdots z_{i_{p}}$ are linearly independent over $C$.

The Killing form induces a natural isomorphism $E_{p} \simeq E_{p}^{*}$ of $G$-modules and hence a natural isomorphism $S / F \otimes E_{p} \simeq \operatorname{Hom}\left(E_{p}, S / F\right)$ of $G$-modules. Under this isomorphism the invariants $I\left(S / F \otimes E_{p}\right)$ correspond to $\operatorname{Hom}_{G}\left(E_{p}, S / F\right)$. For $(i) \in \Omega$, let $\phi_{(i)}$ be the image in $\operatorname{Hom}_{G}\left(E_{p}, S / F\right)$ of $z_{i_{1}} \cdots z_{i_{p}}$. The linear independence of the $z_{i_{1}} \cdots z_{i}$ implies linear independence of the $\phi_{(i)}$. Now if $M$ is an irreducible $G$-module and $\phi_{1}, \cdots, \phi_{s}$ are linearly independent $G$-module homomorphisms of $M$ into a $G$-module $N$ then the sum $\sum_{i} \phi_{i}(M)$ is direct. This follows at once from Schur's lemma by induction on the number of summands. In the case at hand this means that the sum $\Sigma_{(i)} \phi_{(i)}\left(E_{p}\right)$ is direct. Since the number of summands is $\left(\begin{array}{l}n \\ p\end{array}\right)=\operatorname{dim} E_{p}$, the sum $\sum_{(i)} \phi_{(i)}\left(E_{p}\right)$ is the isotypic component of $S / F$ of type $E_{p}$. Thus $E_{p}$ occurs as an irreducible constituent of $(S / F)_{q}$ for precisely the values $q=m_{i_{1}}+\cdots+m_{i_{n}}, i_{1}<\cdots<i_{p}$. From the definition of $\phi_{(i)}$ as the image of $\theta\left(d f_{i_{1}} \cdots d f_{i_{n}}\right)$ it follows that a basis for $\phi_{(i)}\left(E_{p}\right)$ is given by the $\left(\begin{array}{c}n \\ p\end{array}\right)$ minors of $J$ which involve $f_{i_{1}}, \cdots, f_{i_{n}}$, reduced $\bmod F$. This completes the proof of Theorem 2.

For the corollaries we argue as follows:

(2a) The linear independence over $C$ of the minors of $J$ after reduction $\bmod F$ amounts to the linear independence of the elements $\theta\left(d f_{i_{1}} \cdots d f_{i_{n}}\right)$ over $\boldsymbol{C}$. This we have shown.

(2b) The proof of the theorem shows that $I(S / F \otimes E)$ is generated as algebra over $C$ by the $z_{i}=\theta\left(d f_{i}\right)$ and the unit element. Since $z_{i} z_{j}=-z_{j} z_{i}, I(S / F \otimes E)$ is a homomorphic image of an exterior algebra on $n$ generators. But 
$\operatorname{dim} I(S / F \otimes E)=\operatorname{dim} E=2^{n}$ so $I(S / F \otimes E)$ is in fact an exterior algebra on the $z_{i}=\theta\left(d f_{i}\right)$.

(2c) The proof of the theorem shows that $I\left(E_{p} \otimes E_{p}\right)=C w^{p}$ for all $p=1, \cdots, n$ where $w=a u+b v$ may be identified with the Killing form. Suppose we have an isomorphism $E_{p} \simeq E_{q}$ of $G$-modules. Then $\left(\begin{array}{l}n \\ p\end{array}\right)=\operatorname{dim} E_{p}=\operatorname{dim} E_{q}=\left(\begin{array}{l}n \\ q\end{array}\right)$ so $q=p$ or $q=n-p$. Suppose $q=n-p$. Let $\chi_{p}$ be the character of $E_{p}$ and let $\gamma \in G$ be a reflection. Then $\chi_{p}(\gamma)$ is the $p$ th elementary symmetric function of the eigenvalues $1,1, \cdots, 1,-1$ so that $\chi_{p}(\gamma)=\left(\begin{array}{c}n-1 \\ p\end{array}\right)-\left(\begin{array}{c}n-1 \\ p-1\end{array}\right)$. Now $\chi_{p}(\gamma)=\chi_{n-p}(\gamma)$ shows $n-p=p$. Thus in any case $q=p$. It follows that $I\left(E_{p} \otimes E_{q}\right)=0$ for $q \neq p$ and hence $I(E \otimes E)=\Sigma_{p} I\left(E_{p} \otimes E_{p}\right)$ is generated over $C$ by the unit element and an element $w=a u+b v$ which satisfies $w^{n+1}=0$ and which may be identified with the Killing form.

(2d) From Theorem 2 or directly from (5.1) we conclude that $I\left(S / F \otimes E_{p}\right)(t)$ $=\sigma_{p}\left(t^{m_{1}}, \cdots, t^{m_{n}}\right)$. Thus one has a homogeneous isomorphism

$$
I\left(S / F \otimes E_{1}\right) \simeq I\left(S / F \otimes E_{n-1}\right)
$$

of graded vector spaces if and only if there exists an integer $k$ such that $t^{k} \sigma_{1}\left(t^{m_{1}}, \cdots, t^{m_{n}}\right)=\sigma_{n-1}\left(t^{m_{1}}, \cdots, t^{m_{n}}\right)$. Comparing coefficients on both sides shows that this condition is equivalent to the existence of an integer $k$ such that $k+m_{i}+m_{n-i+1}=m_{1}+\cdots+m_{n}$. This is equivalent in turn to the statement that $m_{i}+m_{n-i+1}$ is independent of $i$, the double duality. The same kind of coefficient comparison shows that double duality implies the isomorphisms

$$
I\left(S / F \otimes E_{p}\right) \simeq I\left(S / F \otimes E_{n-p}\right) .
$$

For later use we remark that (5.2) is equivalent to the existence of a homogeneous isomorphism

$$
I\left(S \otimes E_{1}\right) \simeq I\left(S \otimes E_{n-1}\right)
$$

of graded vector spaces.

6. Let $M, N$ be $G$-modules. We say that $M$ and $N$ are skew isomorphic if there exists a 1-1 C-linear map $\theta$ of $M$ onto $N$ such that $\theta \gamma x=(\operatorname{det} \gamma) \gamma \theta x$ for all $x \in M$ and all $\gamma \in G$. We call $\theta$ a skew isomorphism between $M$ and $N$. Since $\operatorname{det} \gamma= \pm 1$ the relation of skew isomorphism is symmetric. Again we let $Z$ denote the 1-dimensional $G$-module defined by the homomorphism $\gamma \rightarrow \operatorname{det} \gamma$ and let $z$ be a generator of $Z$. If we set $\hat{M}=M \otimes Z$ we see that $x \rightarrow x \otimes z, x \in M$, defines a skew isomorphism between $M$ and $\hat{M}$. Since $Z \otimes Z \simeq C, \hat{M}^{\wedge}$ and $M$ are isomorphic as $G$-modules.

From Lemma 1 we conclude that $t^{r}(S / F)\left(t^{-1}, \gamma\right)=\operatorname{det} \gamma(S / F)(t, \gamma)$ where $r=\sum_{i} m_{i}$. Thus $\tau_{r-q}(\gamma)=(\operatorname{det} \gamma) \tau_{q}(\gamma)$ for all $\gamma \in G$ and all $q=0, \cdots, r$ so that $(S / F)_{q}$ and $(S / F)_{r-q}$ are skew isomorphic. If $M$ is an irreducible $G$-module and $N$ 
is any $G$-module, then $\hat{M}$ is irreducible and the multiplicity of $M$ in $N$ is equal to the multiplicity of $\hat{M}$ in $\hat{N}$. Hence the

THEOREM. If $M$ is an irreducible G-module, then the multiplicity of $M$ in $(S / F)_{q}$ is equal to the multiplicity of $\hat{M}$ in $(S / F)_{r-q}$ where $r=\sum_{i} m_{i}$. Thus in the notation of Theorem 1 , with suitable ordering, $q_{i}(\hat{M})=r-q_{i}(M)$.

We say that $x \in M$ is a skew invariant if $\gamma x=(\operatorname{det} \gamma) x$ for all $\gamma \in G$. Let $\hat{I}(M)$ denote the subspace of skew invariant elements of $M$. Then the map $x \rightarrow x \otimes z$ defines a natural isomorphism $\hat{I}(M) \simeq I(\hat{M})$ of vector spaces. It follows that we have an isomorphism

$$
\hat{I}(S \otimes M) \simeq I(S \otimes \hat{M})
$$

of graded vector spaces which is homogeneous of degree zero. From Lemma 2 we see that $I(S \otimes M)$ is free over $I(S)$ of rank equal to $\operatorname{dim} M$.

Since $E_{n-1} \simeq \hat{E}_{1}$ is an isomorphism of $G$-modules, the isomorphism (5.3) equivalent to the double duality becomes

$$
\mathfrak{I}\left(S \otimes E_{1}\right) \simeq I\left(S \otimes E_{1}\right)
$$

Now $I\left(S \otimes E_{1}\right)$ is generated freely over $I(S)$ by the $d f_{i}$. If we can construct the homogeneous derivation $\hat{d}$ of Theorem 3 then $\hat{I}\left(S \otimes E_{1}\right)$ is generated freely over $I(S)$ by the $\hat{d} f_{i}$ and the homogeneous isomorphism (6.2) is defined by $d f_{i} \rightarrow \hat{d} f_{i}$. Thus in this formulation the double duality is equivalent to the existence of the $\operatorname{map} \hat{d}$.

In connection with (6.2) it is worth noting that the homogeneous isomorphism $\hat{I}\left(S \otimes E_{0}\right) \simeq I\left(S \otimes E_{0}\right)$ amounts to the familiar fact that every skew invariant polynomial may be written as an invariant polynomial multiplied by $\operatorname{det} J$.

7. Let $q=m_{1}+\cdots+m_{n-1}$. We have a sequence of natural maps

$$
S_{q} \otimes E_{n-1} \stackrel{\psi_{1}}{\longrightarrow} S_{q} \otimes E_{1}^{*} \stackrel{\psi_{2}}{\longrightarrow} \operatorname{Hom}\left(E_{1}, S_{q}\right) \stackrel{\psi_{3}}{\longrightarrow} \operatorname{Hom}\left(S_{1}, S_{q}\right)
$$

where $\psi_{1}$ is a skew isomorphism of $G$-modules induced by the natural duality in the Grassman algebra, where $\psi_{2}$ is an isomorphism of $G$-modules induced by the natural isomorphism of vector spaces, and $\psi_{3}$ is the isomorphism of $G$-modules induced by the identification of $E_{1}$ with $S_{1}$. The composite map

$$
\psi: S_{q} \otimes E_{n-1} \rightarrow \operatorname{Hom}\left(S_{1}, S_{q}\right)
$$

is a skew isomorphism of $G$-modules. Let $\eta=\psi\left(d f_{1} \cdots d f_{n-1}\right)$. Since $d f_{1} \cdots d f_{n-1} \in I\left(S_{q} \otimes E_{n-1}\right)$ we have $\eta \in \hat{I}\left(\operatorname{Hom}\left(S_{1}, S_{q}\right)\right)$ so that $\eta$ is a skew homomorphism of $S_{1}$ into $S_{q}$. Since $S_{1}$ is irreducible, $\eta$ must be injective, and from the definition of the map $\psi$ we see that $\eta x_{i}=u_{i}$ where $u_{i}$ is the minor of order $n-1$ of $J$ obtained by deleting the derivatives of $f_{n}$ and the derivatives with respect to $x_{i}$. 
Our map $d: S \rightarrow S \otimes E_{1}$ is homogeneous of degree -1 and commutes with the action of $G$. Let $\hat{d}=d \circ \eta$. Then $\hat{d}$ is a skew isomorphism of $S_{1}$ into $S_{q-1} \otimes E_{1}$. Since $S$ is a polynomial ring we may extend $\hat{d}$ to a derivation $\hat{d}: S \rightarrow S \otimes E_{1}$ of $S$-modules. By induction on the degree of a homogeneous element one sees that $\hat{d} \hat{\gamma} s=(\operatorname{det} \gamma) \gamma \hat{d} s$ for all $\gamma \in G$ and all $s \in S$. Thus $\hat{d}$ maps $I(S)$ into $\hat{I}\left(S \otimes E_{1}\right)$. Since $\hat{d} x_{i}=d u_{i}=\Sigma_{k}\left(\partial u_{i} / \partial x_{k}\right) d x_{k}$ we have

$$
\hat{d f}=\sum_{i} \sum_{k} \frac{\partial f}{\partial x_{i}} \frac{\partial u_{i}}{\partial x_{k}} d x_{k}
$$

We claim that the elements $\hat{d} f_{1}, \cdots, \hat{d} f_{n}$ are linearly independent over $S$. If not, then we have a relation $\sum_{i} s_{i} \hat{d} f_{i}=0$ where $s_{i} \in S$ and where $s_{1}$, say, is not zero. Then multiplication by $\hat{d} f_{2} \cdots \hat{d} f_{n}$ shows that $\hat{d} f_{1} \cdots \hat{d} f_{n}=0$. On the other hand, computing directly from (7.1) shows that

$$
\hat{d} f_{1} \cdots \hat{d} f_{n}=\operatorname{det}\left(\frac{\partial f_{i}}{\partial x_{k}}\right) \operatorname{det}\left(\frac{\partial u_{i}}{\partial x_{k}}\right) d x_{1} \cdots d x_{n}
$$

which is not zero in view of our assumption about the algebraic independence of the $u_{i}$. Thus the $\hat{d} f_{i}$ are linearly independent over $S$ and the sum $P=\sum_{i} I(S) \hat{d} f_{i}$ is direct. Since $G$ is a real group it has an invariant quadratic form $f_{1}$ and hence $m_{1}=1$. The degree of the map $\hat{d}$ is thus $q-1=m_{1}+\cdots+m_{n-1}-1=m_{2}+\cdots+m_{n-1}$ and the Poincaré series for the graded vector space $P$ is thus

$$
P(t)=\frac{t^{m_{2}+\cdots+m_{n-1}}\left(t^{m_{1}}+\cdots+t^{m_{n}}\right)}{\left(1-t^{m_{1}+1}\right) \cdots\left(1-t^{m_{n}+1}\right)} .
$$

On the other hand, using (5.1), (6.1) and the double duality we see that $P(t)=I\left(S \otimes E_{n-1}\right)(t)=\hat{I}\left(S \otimes E_{1}\right)(t)$. Since $P \subseteq \hat{I}\left(S \otimes E_{1}\right)$ is an inclusion of graded vector spaces we have $P=\hat{I}\left(S \otimes E_{1}\right)$. Thus $\hat{I}\left(S \otimes E_{1}\right)$ is freely generated over $I(S)$ by $\hat{d} f_{1}, \cdots, \hat{d} f_{n}$ and Theorem 3 is proved.

8. For the symmetric group on $n$ letters $x_{1}, \cdots, x_{n}$ we can give the following construction for the skew invariant differential 1-forms. Let $\sigma_{1}, \cdots, \sigma_{n}$ be the elementary symmetric functions of $x_{1}, \cdots, x_{n}$ and let $\Delta\left(x_{1}, \cdots, x_{n-1}\right)=\prod_{1 \leqq i<j \leqq n-1}\left(x_{i}-x_{j}\right)$ be the fundamental skew invariant polynomial for the symmetric group on the letters $x_{1}, \cdots, x_{n-1}$. Then a basis for the skew invariant differential 1-forms over the algebra of symmetric functions is given by the forms

$$
\omega_{k}=\sum_{i}(-1)^{i+1} \Delta\left(x_{1}, \cdots, \tilde{x}_{i}, \cdots, x_{n}\right) \frac{\partial \sigma_{k}}{\partial x_{i}} d x_{i}, \quad k=1, \cdots, n,
$$

where $\tilde{x}_{i}$ means that the letter $x_{i}$ is to be omitted. 


\section{REFERENCES}

1. W. Burnside, The determination of all groups of rational linear substitutions of finite order which contain the symmetric group in the variables, Proc. London Math. Soc. (2) 10 (1912), 284-308.

2. C. Chevalley, Invariants of finite groups generated by reflections, Amer. J. Math. 77 (1955), 778-782.

3. A. J. Coleman, The Betti numbers of the simple Lie groups, Canad. J. Math. 10 (1958), 349-356.

4. H. S. M. Coxeter, The product of the generators of a finite group generated by reflections, Duke Math. J. 18 (1951), 765-782.

5. J. S. Frame, The classes and representations of the groups of 27 lines and 28 bitangents, Ann. Mat. Pura Appl. 32 (1951), 83-119.

6. G. Frobenius, Uber die Charaktere der symmetrischen Gruppe, S.-B. Preuss. Akad. Wiss. Berlin (1900), 516-534.

7. B. Kostant, The principal three dimensional subgroup and the Betti numbers of a complex simple Lie group, Amer. J. Math. 81 (1959), 973-1032.

8. G. C. Shephard and J. A. Todd, Finite unitary reflection groups, Canad. J. Math. 6 (1954), 274-304.

9. L. Solomon, Invariants of finite reflection groups, Nagoya Math. J. 22 (1963), 57-64.

INSTITUTE FOR ADVANCED STUDY,

Princeton, New Jersey

HAVERFORD COLLEGE,

Haverford, Pennsylvania 